The AstrophysiCAL JourNAL SUPPLEMENT SERIES, 131:375-389, 2000 November

(C) 2000. The American Astronomical Society. All rights reserved. Printed in U.S.A.

\title{
ELECTRON-ION RECOMBINATION RATE COEFFICIENTS AND PHOTOIONIZATION CROSS SECTIONS FOR ASTROPHYSICALLY ABUNDANT ELEMENTS. IV. RELATIVISTIC CALCULATIONS FOR C IV AND C v FOR ULTRAVIOLET AND X-RAY MODELING
}

\author{
Sultana N. Nahar and Anil K. Pradhan \\ Department of Astronomy, Ohio State University, Columbus, OH 43210; pradhan@astronomy.ohio-state.edu \\ AND \\ HoNG LIN ZHANG \\ Applied Theoretical \& Computational Physics Division, MS F663, Los Alamos National Laboratory, Los Alamos, NM 87545 \\ Received 2000 March 15 ; accepted 2000 June 15
}

\begin{abstract}
The first complete set of unified cross sections and rate coefficients are calculated for photoionization and recombination of $\mathrm{He}$ - and Li-like ions using the relativistic Breit-Pauli $\boldsymbol{R}$-matrix method. We present total, unified $(e+$ ion) recombination rate coefficients for $(e+\mathrm{C} \mathrm{vI} \rightarrow \mathrm{C} \mathrm{v})$ and $(e+\mathrm{C} \mathrm{v} \rightarrow \mathrm{C} \mathrm{IV})$, including fine structure. Level-specific recombination rate coefficients up to the $n=10$ levels are also obtained for the first time; these differ considerably from the approximate rates currently available. Applications to recombination-cascade coefficients in X-ray spectral models of $\mathrm{K} \alpha$ emission from the important He-like ions are pointed out. The overall uncertainty in the total recombination rates should not exceed $10 \%-20 \%$. Ionization fractions for carbon are recomputed in the coronal approximation using the new rates. The present $(e+$ ion) recombination rate coefficients are compared with several sets of available data, including previous $L S$ coupling results and "experimentally derived" rate coefficients. The roles of relativistic fine structure, resolution of resonances, radiation damping, and interference effects are discussed. Two general features of recombination rates are noted: (1) the nonresonant (radiative recombination) peak as $E, T \rightarrow 0$, and (2) the high- $T$ resonant (dielectronic recombination) peak.

Subject headings: atomic data — atomic processes — line: formation $-\mathrm{X}$-rays: general
\end{abstract}

\section{INTRODUCTION}

Electron-ion recombination with $\mathrm{H}$ - and $\mathrm{He}$-like ions is of particular interest in X-ray astronomy. ${ }^{1} \mathrm{X}$-ray emission in the $\mathrm{K} \alpha$ complex of He-like ions, such as $\mathrm{C} \mathrm{v}$, from the $n=2 \rightarrow 1$ transitions yields perhaps the most useful spectral diagnostics for temperature, density, ionization balance, and abundances in the plasma source (Gabriel 1972; Mewe \& Schrijver 1978; Pradhan \& Shull 1981; Pradhan 1985). Li-like C IV is of considerable importance in UV emission spectra from active galactic nuclei (AGNs) and quasars (e.g., Laor et al. 1994), as well as absorption in AGN (Crenshaw \& Kraemer 1999). In addition, the C IV and other Li-like ionization states are valuable tracers of the plasma in the "hot interstellar medium" (Spitzer 1990; Spitzer \& Fitzpatrick 1993; Martin \& Bowyer 1990; Bregman \& Harrington 1986). The primary sets of atomic data needed for accurate calculations of ionization fractions are for photoionization and recombination.

Theoretical models of spectral formation also require excitation cross sections and transition probabilities. A considerable amount of atomic data is being computed for these atomic processes under the Iron Project (IP; Hummer et al. 1993) for electron impact excitation and radiative transition probabilities for astrophysically abundant elements using the Breit-Pauli $\boldsymbol{R}$-matrix (BPRM) method that includes relativistic fine structure in intermediate coupling

\footnotetext{
${ }^{1}$ The proceedings of the workshop on "Atomic Data Needs in X-ray Astronomy," held at the Goddard Space Flight Center, Maryland, 1999 December 17-18, can be found at http://heasarc.gsfc.nasa.gov.
}

(Berrington, Eissner, \& Norrington 1995). The present work is an extension of the IP work to photoionization and recombination.

The ionization balance equations usually correspond to photoionization equilibrium

$$
\begin{aligned}
\int_{v_{0}}^{\infty} \frac{4 \pi J_{v}}{h v} N\left(X^{z}\right) \sigma_{\mathrm{PI}}\left(v, X^{z}\right) d v \\
\quad=\sum_{j} N_{e} N\left(X^{z+1}\right) \alpha_{R}\left(X_{j}^{z} ; T\right),
\end{aligned}
$$

and collisional equilibrium

$$
C_{I}\left(T, X^{z}\right) N_{e} N\left(X^{z}\right)=\sum_{j} N_{e} N\left(X^{z+1}\right) \alpha_{R}\left(X_{j}^{z} ; T\right),
$$

where the $\sigma_{\mathrm{PI}}$ are the photoionization cross sections, and the $\alpha_{R}\left(X_{j}^{z} ; T\right)$ are the total electron-ion recombination rate coefficients of the recombined ion of charge $z, X_{j}^{z}$, to state $j$ at electron temperature $T$. The $C_{I}$ are the rate coefficients for electron impact ionization that can be reliably obtained from experimental measurements (Bell et al. 1983). On the other hand, the $(e+$ ion $)$ recombination cross sections and rates are difficult to compute or measure. However, several experimental measurements of electron-ion recombination cross sections using ion storage rings have been carried out in recent years (e.g., Wolf et al. 1991; Kilgus et al. 1990, 1993; Mannervik et al. 1997). The experimental cross sections exhibit detailed resonance structures observed at very high resolution in beam energy and measure absolute cross sections. Therefore, they provide ideal tests for theoretical methods, as well as the physical effects included in 
the calculations. Many of these experimental measurements have been for recombination with $\mathrm{H}-$ and $\mathrm{He}-$ like $\mathrm{C}$ and $\mathrm{O}$.

Among the recent theoretical developments is a selfconsistent method for calculations for photoionization and $(e+$ ion) recombination, as described in previous papers in this series. An identical eigenfunction expansion for the ion is employed in coupled channel calculations for both processes, thus ensuring consistently accurate cross sections and rates in an ab initio manner. The theoretical treatment of $(e+$ ion $)$ recombination subsumes both the nonresonant recombination (i.e., radiative recombination, RR) and the resonant recombination (i.e., dielectronic recombination, DR) processes in a unified scheme. In addition to the total, unified recombination rates, level-specific recombination rates and photoionization cross sections are obtained for a large number of atomic levels. The calculations are carried out in the close coupling approximation using the $\boldsymbol{R}$-matrix method. Although the calculations are computationally intensive, they yield nearly all photoionization and recombination parameters needed for astrophysical photoionization models with higher precision than hitherto possible.

Previous calculations of unified $(e+$ ion) recombination cross sections and rates, reported in the present series on photoionization and recombination, were carried out in $L S$ coupling (Nahar \& Pradhan 1997, hereafter cited as Paper I; Nahar 1999). There were two reasons. First, the calculations are extremely complex and involve both radiative photoionization and collisional electron-ion scattering calculations; the full intermediate coupling relativistic calculations are many times more computationally intensive than the $L S$ coupling ones. Second, the effect of relativistic fine structure was expected to be small for these light elements.

For the highly charged $\mathrm{H}$ - and He-like ions, however, subsequent calculations showed that results including the relativistic effects are significantly more accurate not only in terms of more detailed resonance structure but also to enable a full resolution of resonances necessary to include radiative damping (Pradhan \& Zhang 1997; Zhang, Nahar, \& Pradhan 1999, and references therein). The relativistic Breit-Pauli $\boldsymbol{R}$-matrix (BPRM) method is now extended to calculate the total and level-specific recombination rate coefficients in the self-consistent unified manner. This paper describes the first of a series of full-scale BPRM calculations of photoionization and photorecombination, as inverse processes, and DR, to obtain total, unified $(e+$ ion) recombination rates of $\mathrm{He}$ - and Li-like carbon, $\mathrm{C}$ IV and $\mathrm{C} \mathrm{V}$.

\section{THEORY}

The extension of the close-coupling method to electronion recombination is described in earlier works (Nahar \& Pradhan 1994, 1995) together with the details of the unified treatment. Here we present a brief description of the theory relevant to the calculations of electron recombination cross sections with $\mathrm{H}$-like and He-like ions. The calculations are carried out in the close-coupling (CC) approximation employing the $\boldsymbol{R}$-matrix method in intermediate coupling with the BP Hamiltonian. The target ion is represented by an $N$-electron system, and the total wave function expansion, $\Psi(E)$, of the $(N+1)$ electron-ion system of symmetry $S L \pi$ or $J \pi$ may be represented in terms of the target eigenfunctions as

$$
\Psi(E)=A \sum_{i} \chi_{i} \theta_{i}+\sum_{j} c_{j} \Phi_{j}
$$

where $\chi_{i}$ is the target wave function in a specific state $S L \pi$ or level $J_{i} \pi_{i}$, and $\theta_{i}$ is the wave function for the $(N+1)$-th electron in a channel labeled as $S_{i} L_{i}\left(J_{i}\right) \pi_{i} k_{i}^{2} \ell_{i}(J \pi) ; k_{i}^{2}$ being its incident kinetic energy. $\Phi_{j}$ 's are the correlation functions of the $(N+1)$-electron system that account for short-range correlation and the orthogonality between the continuum and the bound orbitals.

In the relativistic BPRM calculations, the set of $S L \pi$ are recoupled to obtain $(e+$ ion) levels with total $J \pi$, followed by diagonalization of the $(N+1)$-electron Hamiltonian,

$$
H_{N+1}^{B P} \Psi=E \Psi \text {. }
$$

The BP Hamiltonian is

$$
H_{N+1}^{\mathrm{BP}}=H_{N+1}+H_{N+1}^{\mathrm{mass}}+H_{N+1}^{\mathrm{Dar}}+H_{N+1}^{\mathrm{so}},
$$

where $H_{N+1}$ is the nonrelativistic Hamiltonian,

$$
H_{N+1}=\sum_{i=1}^{N+1}\left\{-\nabla_{i}^{2}-\frac{2 Z}{r_{i}}+\sum_{j>i}^{N+1} \frac{2}{r_{i j}}\right\},
$$

and the additional terms are the one-body terms, the masscorrection term, the Darwin term, and the spin-orbit term, respectively. Spin-orbit interaction, $H_{N+1}^{\text {so }}$, splits the $L S$ terms into fine-structure levels labeled by $J \pi$, where $J$ is the total angular momentum.

The positive and negative energy states (eq. [4]) define continuum or bound $(e+$ ion) states.

$$
\begin{aligned}
& E=k^{2}>0 \rightarrow \text { continuum (scattering) channels, } \\
& E=-\frac{z^{2}}{v^{2}}<0 \rightarrow \text { bound states }
\end{aligned}
$$

where $v$ is the effective quantum number relative to the core level. If $E<0$, then all continuum channels are "closed" and the solutions represent bound states.

The photoionization cross section can be obtained as

$$
\sigma_{\mathrm{PI}}=\frac{1}{g} \frac{4 \pi^{2}}{3 c} \omega S
$$

where $g$ is the statistical weight factor of the bound state, $S$ is the dipole line strength,

$$
S=\left|\left\langle\Psi_{B}|| D|| \Psi_{F}\right\rangle\right|^{2},
$$

and $\boldsymbol{D}$ is the dipole operator (e.g., Seaton 1987).

For highly charged ions (such as the $\mathrm{H}$ - and the He-like ions), radiative transition probabilities in the core ion are very large and may be of the same order of magnitude as autoionization probabilities. Autoionizing resonances may then undergo significant radiative decay and the photoionization process may be written as

$$
h v+X^{+} \rightarrow\left(X^{+}\right)^{* *} \rightarrow\left\{\begin{array}{l}
(i) e+X^{++} \\
\text {(ii) } h v^{\prime}+X^{+}
\end{array}\right\} .
$$

Branch (ii) represents radiation damping of autoionizing resonances. In the present work, this radiative damping effect is included for all near-threshold resonances, up to $v \leq 10$, using a resonance fitting procedure (Sakimoto, Terao, \& Berrington 1990; Pradhan \& Zhang 1997; Zhang et al. 1999).

Recombination of an incoming electron to the target ion may occur through nonresonant, background continuum, usually referred to as radiative recombination (RR),

$$
e+X^{++} \rightarrow h v+X^{+},
$$


which is the inverse process of direct photoionization, or through the two-step recombination process via autoionizing resonances, i.e., dielectronic recombination (DR):

$$
e+X^{++} \rightarrow\left(X^{+}\right)^{* *} \rightarrow\left\{\begin{array}{l}
(i) e+X^{++} \\
\text {(ii) } h v+X^{+}
\end{array}\right\},
$$

where the incident electron is in a quasi-bound doubly excited state, which leads either to (i) autoionization, a radiation-less transition to a lower state of the ion and the free electron, or to (ii) radiative stabilization to recombining ion states predominantly via decay of the ion core (usually to the ground state) and the bound electron.

In the unified treatment, the photoionization cross sections, $\sigma_{\mathrm{PI}}$, of a large number of low- $n$ bound states-all possible states with $n \leq n_{\max } \sim 10$-are obtained in the CC approximation as in the Opacity Project (Seaton 1987). Coupled channel calculations for $\sigma_{\mathrm{PI}}$ include both the background and the resonance structures (caused by the doubly excited autoionizing states) in the cross sections. The recombination cross section, $\sigma_{\mathrm{RC}}$, is related to $\sigma_{\mathrm{PI}}$ through detailed balance (Milne relation) as

$$
\sigma_{\mathrm{RC}}(\epsilon)=\frac{\alpha^{2}}{4} \frac{g_{i}}{g_{j}} \frac{(\epsilon+I)^{2}}{\epsilon} \sigma_{\mathrm{PI}}
$$

in rydberg units; $\alpha$ is the fine-structure constant, $\epsilon$ is the photoelectron energy, and $I$ is the ionization potential. In this work, it is assumed that the recombining ion is in the ground state and that recombination can take place into the ground or any of the excited recombined $(e+$ ion) states. Recombination rate coefficients of individual levels are obtained by averaging the recombination cross sections over the Maxwellian electron distribution, $f(v)$, at a given temperature as

$$
\alpha_{\mathrm{RC}}(T)=\int_{0}^{\infty} v f(v) \sigma_{\mathrm{RC}} d v .
$$

The contributions of these bound states to the total $\sigma_{\mathrm{RC}}$ are obtained by summing over the contributions from individual cross sections. $\sigma_{\mathrm{RC}}$ thus obtained from $\sigma_{\mathrm{PI}}$, including the radiatively damped autoionizing resonances (eq. [10]), corresponds to the total (DR + RR) unified recombination cross section.

The recombination cross section, $\sigma_{\mathrm{RC}}$, in megabarns $(\mathrm{Mb})$, is related to the collision strength, $\Omega_{\mathrm{RC}}$, as

$$
\sigma_{\mathrm{RC}}(i \rightarrow j)(\mathrm{Mb})=\pi \Omega_{\mathrm{RC}}(i, j) /\left(g_{i} k_{i}^{2}\right)\left(a_{o}^{2} / 1 . \times 10^{-18}\right),
$$

where $k_{i}^{2}$ is the incident electron energy in rydbergs. As $\sigma_{\mathrm{RC}}$ diverges at zero-photoelectron energy, the total collision strength, $\Omega$, is used in the recombination rate calculations.

Recombination into the high- $n$ states must also be included, i.e., $n_{\max }<n \leq \infty$, (Fig. 1 of Nahar \& Pradhan 1994). To each excited threshold $S_{i} L_{i}\left(J_{i}\right) \pi_{i}$ of the $N$-electron target ion, there corresponds an infinite series of $(N+1)$ electron states, $S_{i} L_{i}\left(J_{i}\right) \pi_{i} v \ell$, to which recombination can occur, where $v$ is the effective quantum number. For these states, DR dominates the recombination process and the background recombination is negligibly small. The contributions from these states are added by calculating the collision strengths, $\Omega_{\mathrm{DR}}$, employing the precise theory of radiation damping by Bell \& Seaton (1985) and Nahar \& Pradhan (1994). Several aspects related to the application of the theory to the calculation of DR collision strengths are described in the references cited. General details of the theory and close-coupling BPRM calculations are described in Paper I and in Zhang et al. (1999, and references therein).

\section{COMPUTATIONS}

The electron-ion recombination calculations entail CC calculations for photoionization and electron-ion scattering. Identical eigenfunction expansion for the target (core) ion is employed for both processes, thus enabling inherently self-consistent photoionization/recombination results in an ab initio manner for a given ion. The total recombination cross sections, $\sigma_{\mathrm{RC}}$, are obtained from the photoionization cross sections, $\sigma_{\mathrm{PI}}$, and DR collision strengths, $\Omega_{\mathrm{DR}}$, are calculated as described in Paper I and in Zhang et al. (1999). However, the computations for the cross sections are repeated with a much finer energy mesh in order to delineate the detailed resonance structures as observed in the experiments.

Computations of photoionization cross sections, $\sigma_{\mathrm{PI}}$, in the relativistic BPRM intermediate coupling approximations are carried out using the package of codes from the Iron Project (Berrington et al. 1995; Hummer et al. 1993). However, radiation damping of resonances up to $n=10$ are included through use of the extended codes of STGF and STGBF (Pradhan \& Zhang 1997). The $R$-matrix calculations are carried out for each total angular momentum symmetry, $J \pi$, corresponding to a set of fine-structure target levels, $J_{t}$.

In the energy region from threshold up to about $v=$ $v_{\max }=10(v$ is the effective quantum number of the outer orbital of the recombined ion bound state), detailed photorecombination cross sections are calculated as in equation (12). The electrons in this energy range recombine to a large number of final $(e+$ ion) states and recombination cross sections are computed for all coupled symmetries and summed to obtain the total $\sigma_{\mathrm{RC}}$. The number of these final recombined states in the BPRM case is larger, owing to more channels involving fine structure, than in the $L S$ coupling case.

In the higher energy region, $v_{\max }<v<\infty$ below each threshold target level, where the resonances are narrow and dense and the background is negligible, we compute the detailed and the resonance-averaged DR cross sections. The

TABLE 1

Target Terms in the Eigenfunction Expansions of C V AND C VI WITH TARGET ENERGIES IN eV

\begin{tabular}{lc|cr}
\hline \hline \multicolumn{2}{c|}{$\mathrm{C} \mathrm{v}^{\mathrm{a}}$} & \multicolumn{2}{|c}{$\mathrm{C} \mathrm{vI}^{\mathrm{b}}$} \\
\hline $1 s^{2}\left({ }^{1} S_{0}\right) \ldots \ldots \ldots$ & 0.0 & $1 s\left({ }^{2} S_{1 / 2}\right) \ldots \ldots$ & 0.00 \\
$1 s 2 s\left({ }^{3} S_{1}\right) \ldots \ldots$ & 298.73 & $2 s\left({ }^{2} S_{1 / 2}\right) \ldots \ldots$ & 367.36 \\
$1 s 2 s\left({ }^{1} S_{0}\right) \ldots \ldots$ & 304.38 & $2 p\left({ }^{2} P_{1 / 2}\right) \ldots \ldots$ & 367.36 \\
$1 s 2 p\left({ }^{3} P_{0}\right) \ldots \ldots$ & 304.39 & $2 p\left({ }^{2} P_{3 / 2}\right) \ldots \ldots$ & 367.42 \\
$1 s 2 p\left({ }^{3} P_{1}\right) \ldots \ldots$ & 304.39 & $3 s\left({ }^{2} S_{1 / 2}\right) \ldots \ldots$ & 435.41 \\
$1 s 2 p\left({ }^{3} P_{2}\right) \ldots \ldots$ & 304.41 & $3 p\left({ }^{2} P_{1 / 2}\right) \ldots \ldots$ & 435.41 \\
$1 s 2 p\left({ }^{1} P_{1}\right) \ldots \ldots$ & 307.90 & $3 p\left({ }^{2} P_{3 / 2}\right) \ldots \ldots$ & 435.43 \\
$1 s 3 s\left({ }^{3} S_{1}\right) \ldots \ldots$ & 352.05 & $3 d\left({ }^{2} D_{3 / 2}\right) \ldots \ldots$ & 435.43 \\
$1 s 3 s\left({ }^{1} S_{0}\right) \ldots \ldots$ & 353.49 & $3 d\left({ }^{2} D_{5 / 2}\right) \ldots \ldots$ & 435.43 \\
$1 s 3 p\left({ }^{3} P_{0}\right) \ldots \ldots$ & 353.52 & $\ldots$ & $\ldots$ \\
$1 s 3 p\left({ }^{3} P_{1}\right) \ldots \ldots$ & 353.52 & $\ldots$ & $\ldots$ \\
$1 s 3 p\left({ }^{3} P_{2}\right) \ldots \ldots$ & 353.52 & $\ldots$ & $\ldots$ \\
$1 s 3 p\left({ }^{1} P_{0}\right) \ldots \ldots$ & 354.51 & $\ldots$ & $\ldots$ \\
\hline
\end{tabular}

a 13-CC, 13-level close-coupling expansion.

b 9-CC, 9-level close-coupling expansion. 
DR collision strengths in BPRM are obtained using extensions of the $\boldsymbol{R}$-matrix asymptotic region codes (Paper I; Zhang \& Pradhan 1997), respectively. It is necessary to use extremely fine energy meshes to delineate the resonance structures belonging to each $n$-complex.

The level-specific recombination-rate coefficients are obtained using a new computer program, BPRRC (Nahar $\&$ Pradhan 2000). The program extends the photoionization cross sections at the high-energy region, beyond the highest target threshold in the $\mathrm{CC}$ wave function expansion of the ion, by a tail from Kramers's fit of $\sigma_{\mathrm{PI}}(E)=\sigma_{\mathrm{PI}}^{o}\left(E_{o}^{3} / E^{3}\right)$. The level-specific rates are obtained for energies going up to infinity. These rates include both nonresonant and resonant contributions up to energies $z^{2} / v_{\max }^{2}$; contributions from all autoionizing resonances up to $v \leq v_{\max } \approx 10$ are included.
The program BPRRC sums the level-specific rates, the result of which is added to the contributions from the high- $n$ DR to obtain the total recombination rates. As an additional check on the numerical calculations, the total recombination-rate coefficients, $\alpha_{R}$, are also calculated from the total recombination collision strength obtained from all the photoionization cross sections and the DR collision strengths. The agreement between the two numerical approaches is within a few percent.

The background contribution from the high- $n$ states $(10<n \leq \infty)$ to the total recombination is also included as the "top-up" part (Nahar 1996). This contribution is important at low temperatures but is negligible at high temperatures. The rapid rise in $\alpha_{R}$ toward very low temperatures is caused by low-energy recombination into the

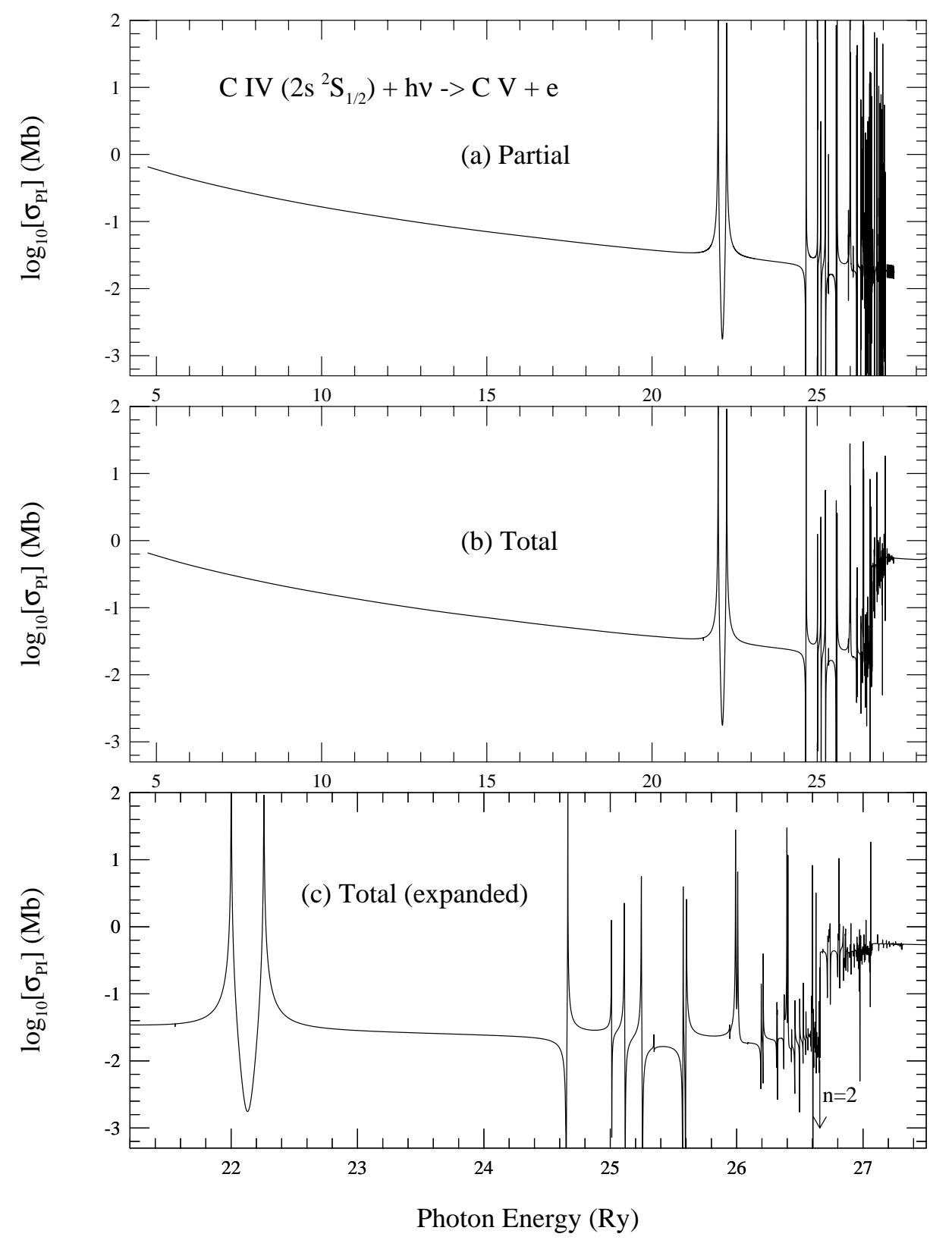

FIG. 1.-Photoionization of the ground state $1 s^{2} 2 s\left({ }^{2} S_{1 / 2}\right)$ of $\mathrm{C}$ IV: partial cross section into the ground level $1 s^{2}\left({ }^{1} S_{0}\right)$ of $\mathrm{C} \mathrm{v}(b)$; total cross section $(a)$; an expanded view of the resonances and inner-shell thresholds $(c)$. The large jump in $(b)$ corresponds to the K-shell ionization edge. (The total cross sections are at a coarser mesh than the partial ones, therefore some of the resonance heights are smaller.) 
infinite number of these high- $n$ states at electron energies not usually high enough for resonant excitations.

The following subsections describe the calculations individually for the ions under consideration.

$$
\text { 3.1. } e+\mathrm{C} \mathrm{V} \rightarrow \mathrm{C} \text { IV }
$$

The fine-structure levels of the target ion, $\mathrm{C} \mathrm{V}$, included in the wave function expansion for $\mathrm{C} \mathrm{IV}$, are given in Table 1. The 13 fine-structure levels of $\mathrm{C} \mathrm{V}$ up to $3 p$ correspond to configurations $1 s^{2}, 1 s 2 s, 1 s 2 p, 1 s 3 s, 1 s 3 p$ (correlation configurations also involve the $n=4$ orbitals). Although calculated energies are within a few percent of the observed ones, the latter are used in the computations for more accurate positions of the resonances. The bound channel wave function, the second term in $\Psi$ in equation (3), contains configuration $3 d^{2}$. Levels of angular momentum symmetries
$1 / 2 \leq J \leq 9 / 2$ are considered. With largest partial wave of the outer electron as $l=9$, these correspond to $0 \leq L \leq 7$ in doublet and quartet spin symmetries. The $\boldsymbol{R}$-matrix basis set is represented by 30 continuum functions. It is necessary to represent the wave function expansion in the inner region of the $\boldsymbol{R}$-matrix boundary with a relatively large number of terms to avoid some numerical problems that result in slight oscillation in computed cross sections.

$$
\text { 3.2. } e+\mathrm{C} \mathrm{VI} \rightarrow \mathrm{C} \mathrm{V}
$$

The wave function expansion of $\mathrm{C} \mathrm{V}$ is represented by nine fine-structure levels (Table 1 ) of hydrogenic $\mathrm{C}$ vi from $1 s$ to $3 d$. Correlation orbitals $4 s, 4 p, 4 d$, and $4 f$ are also included. The $S L \pi$ symmetries consist of $0 \leq L \leq 7$ of singlet and triplet spin symmetries for even and odd parities. All levels of $\mathrm{C} \mathrm{v}$ with total angular momentum
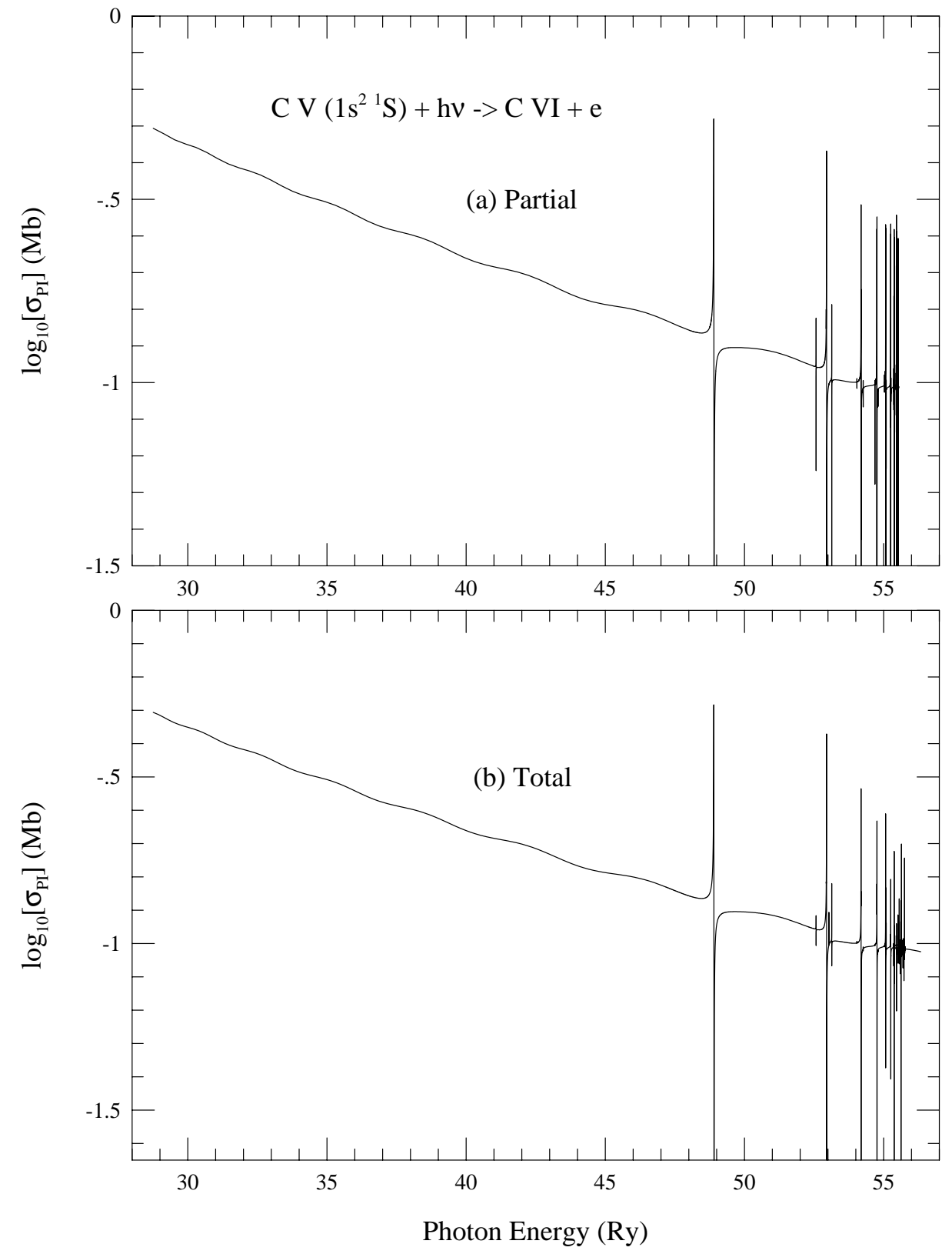

FIG. 2.-Photoionization of the ground state $1 s^{2}\left({ }^{1} S_{0}\right)$ of $\mathrm{C} \mathrm{v}$ : partial cross section into the ground level $1 s\left({ }^{2} S_{1 / 2}\right)$ of C VI $(a)$; total cross section $(b)$. (The total cross sections are at a coarser mesh than the partial ones, therefore some of the resonance heights are smaller.) 

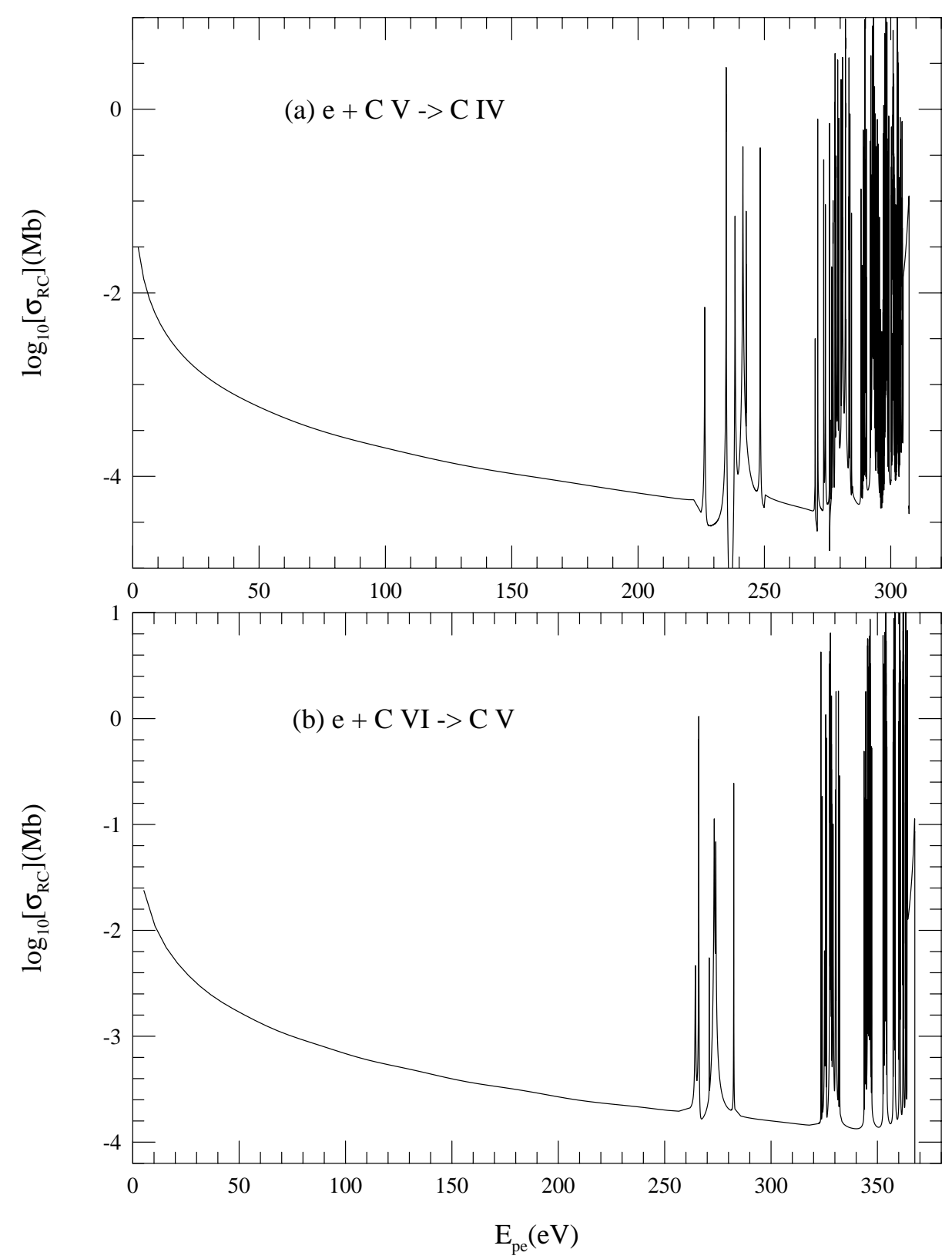

FIG. 3.-Total unified $\left(e+\right.$ ion) photorecombination cross sections, $\sigma_{\mathrm{RC}}$, of $(a) \mathrm{C} \mathrm{IV}$ and $(b) \mathrm{C}$ v. Note that the $\sigma_{\mathrm{RC}}$ exhibit more resonance structures than the corresponding ground level $\sigma_{\mathrm{PI}}$ in Figs. 1 and 2 since the former are summed over the ground and many excited recombined levels.

symmetry $0 \leq J \leq 6$ are included. The $R$-matrix basis set consists of 40 terms to reduce numerical instabilities that might otherwise result in slight oscillations in the cross sections.

\section{RESULTS AND DISCUSSION}

Results for photoionization and recombination are presented below, followed by a discussion of the physical features and effects.

\subsection{Photoionization}

The ground-state cross sections are needed for various astrophysical models, such as in the determination of the ionization fraction in photoionization equilibrium of plasma. Figures 1 and 2 present the ground-state photoionization cross section for C IV $\left(1 s^{2} 2 s\left({ }^{2} S_{1 / 2}\right)\right)$ and $\mathrm{C} \mathrm{V}$ $\left(1 s^{2}\left({ }^{1} S_{0}\right)\right)$. Plots $(a)$ and $(b)$ in each figure show the partial cross section $(a)$ and the total cross section $(b)$ into the ground level of the residual ion. The total cross sections (Figs. $1 b$ and $2 b$ show the K-shell ionization jump at the $n=2$ target levels, i.e., inner-shell photoionization

$$
h v+\mathrm{C} \operatorname{Iv}\left(1 s^{2} 2 s, 2 p\right) \rightarrow e+1 s 2 s(, 2 p),
$$

and

$$
h v+\mathrm{C} \mathrm{v}\left(1 s^{2}, 1 s 2 p\right) \rightarrow e+2 s(, 2 p) .
$$

In X-ray photoionization models, these inner-shell edges play an important role in the overall ionization efficiency.

For both C IV and C v, the first excited target $n=2$ threshold(s) lie at a high energy and the cross sections show a monotonic decrease over a relatively large energy range. (Slight oscillations are seen in the ground level of $\mathrm{C} \mathrm{v}$ because of some numerical instability; as mentioned earlier, such oscillations are reduced using a larger $\boldsymbol{R}$-matrix basis 


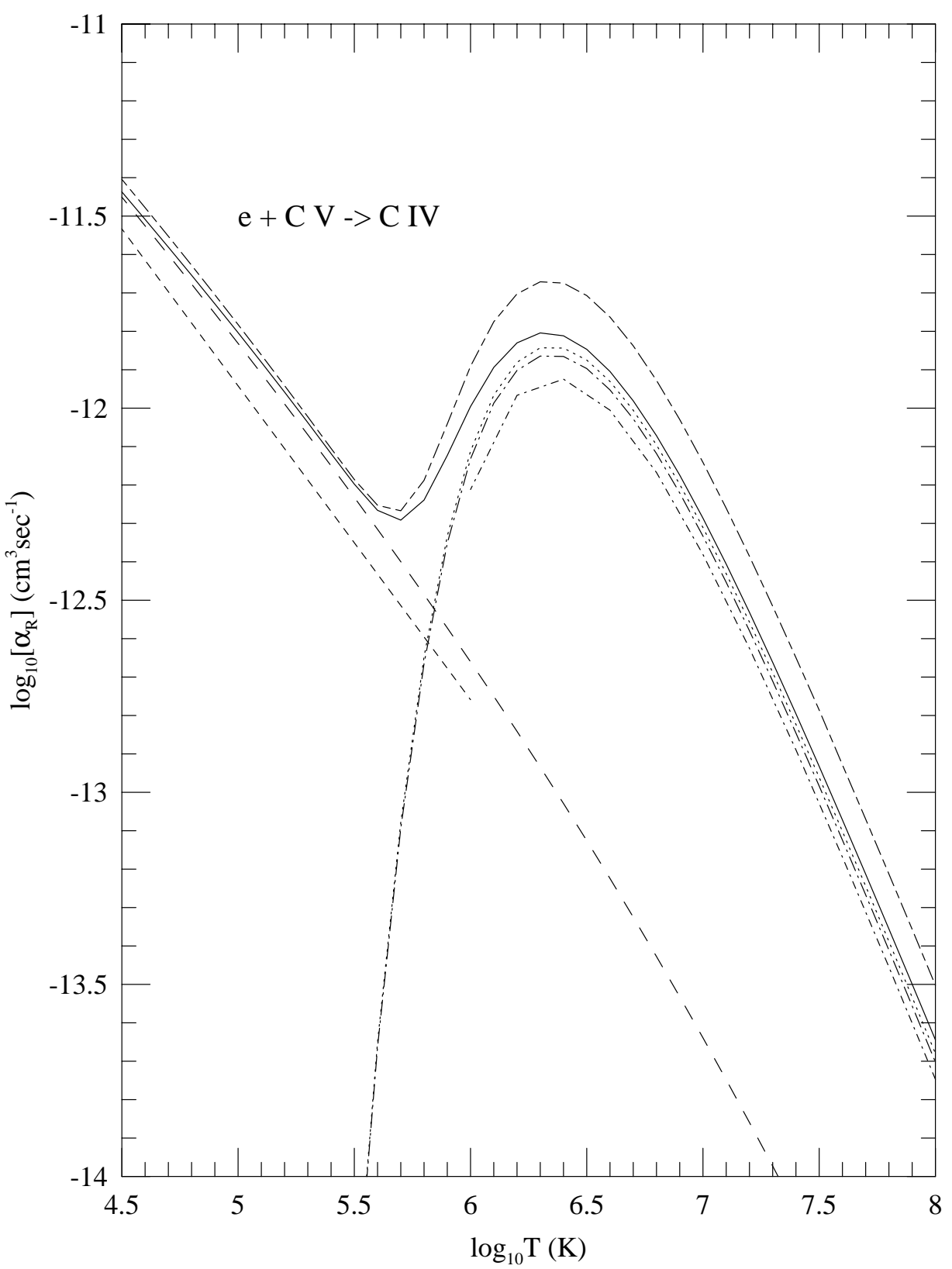

FIG. 4. - $(e+\mathrm{C} \mathrm{v}) \rightarrow \mathrm{C}$ Iv; total unified rate coefficients: BPRM (with fine structure), solid curve; $L S$ coupling, short- and long-dashed curve; from Zhang et al (1999), dotted; from Savin (1999), dotted-long-dashed curve; LS coupling DR rates from Badnell et al (1990), dot-dashed curve; RR rates from Aldrovandi \& Pequignot (1973), short-dashed curve; RR rates from Verner \& Ferland (1996), long-dashed curve.

set.) The resonances at high energies belong to a Rydberg series of $n=2,3$ levels.

\section{RECOMBINATION CROSS SECTIONS AND RATE COEFFICIENTS}

Figures $3 a$ and $3 b$ present the total recombination cross sections, $\sigma_{\mathrm{RC}}$, for $\mathrm{C}$ IV and $\mathrm{C} \mathrm{V}$. In contrast to the earlier presentation for the small energy range (Zhang et al. 1999) to compare with experiments, the figures display the cross sections from threshold to the energy of the highest target threshold, $3 d$, considered in the present work. The resonances belong to different $n$-complexes; these are in close agreement with experimental data (Zhang et al. 1999).

Figure 4 presents total unified recombination rate coefficients for $e+\mathrm{C} \mathrm{V} \rightarrow \mathrm{C}$ IV. The solid curve is the present $\alpha_{R}$ in relativistic BP approximation, and the short-long dashed line represents earlier unified rates in $L S$ coupling and where radiation damping effect was not included (Nahar \& Pradhan 1997). In the high-temperature region, the earlier $L S$ rates significantly overestimate the recombination rate. We compare the present BPRM rates with several other available sets of data, e.g., "experimentally derived" DR rates (dot-long-dashed curve, Savin 1999; which in fact include both the RR + DR contribution - see below) and previous theoretical DR rate coefficients in $L S$ coupling (dot-dashed curve, Badnell et al. 1990).

Zhang et al. (1999) compared in detail the BPRM cross sections with experimental data from ion storage rings for $e+\mathrm{C} \mathrm{V} \rightarrow \mathrm{C}$ IV, with close agreement in the entire range of measurements for both the background (nonresonant) cross sections and resonances. The reported experimental data is primarily in the region of low-energy resonances that domi- 


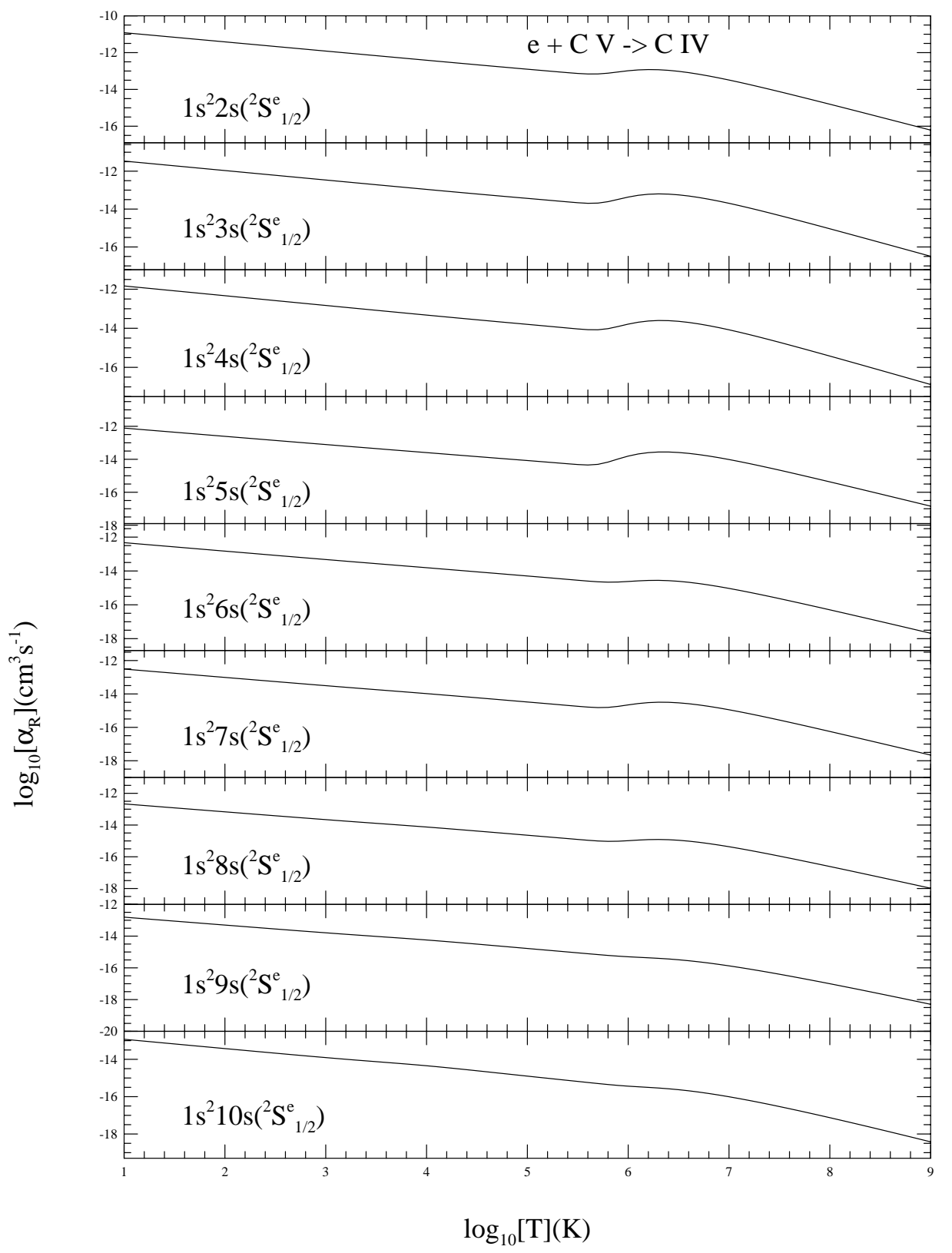

FIG. 5.-Level-specific recombination-rate coefficients of $e+\mathrm{C} \mathrm{v} \rightarrow \mathrm{C}$ IV into the ground and excited levels of the $1 s^{2} n s$ Rydberg series, $n \leq 10$

nate recombination (mainly DR) with $\mathrm{H}$ - and He-like ions. The recombination rate coefficients, $\alpha_{R}$, obtained using the cross sections calculated by Zhang et al. (dotted curve) agree closely with those of Savin (1999) (dotted-long-dashed curve), who used the experimental cross sections to obtain "experimentally derived DR rates." However, these rates do not include contributions from much of the low-energy nonresonant RR and very high energy regions. The total unified $\alpha_{R}(T)$ (solid curve), which include all possible contributions, is, therefore, somewhat higher than that obtained from a limited energy range. The $L S$ coupling DR rate by Badnell et al. (1990) (dot-dashed curve) is lower than the others. The dashed and the long-dashed curves in the figure are RR rates by Aldrovandi \& Pequignot (1973) and Verner $\&$ Ferland (1996); the latter agrees with the present rates at lower temperatures.

In Figures 5 and 6, we show the level-specific recombination rate coefficients into the lowest, and the excited, bound levels of $\mathrm{C}$ IV, for the $1 s^{2} n s, n p$ Rydberg series up to $n=10$. These are the first such calculations; level-specific data have been obtained for all $\ell \leq 9$ and associated $J \pi$ symmetries. The behavior of the level-specific rates mimics that of the total (this is true only for the simple systems under consideration; in general, the level-specific rates show significantly different structure for complex ions, as seen in our previous works). The only distinguishing feature is the DR bump. Since the numerical computations are enormously involved, particularly related to the resolution of resonances, the absence of any unidentified features in the level-specific rates is reassuring.

Total $\alpha_{R}(T)$ for $e+\mathrm{C}$ VI $\rightarrow \mathrm{C}$ v are given in Table 2 and are plotted in Figure 7. The total unified recombination rate coefficients in the present BPRM calculations are plotted in the solid curve. The short-long-dashed curve represents earlier rates obtained in $L S$ coupling and with no inclusion of radiation damping of low- $n$ autoionizing resonances 


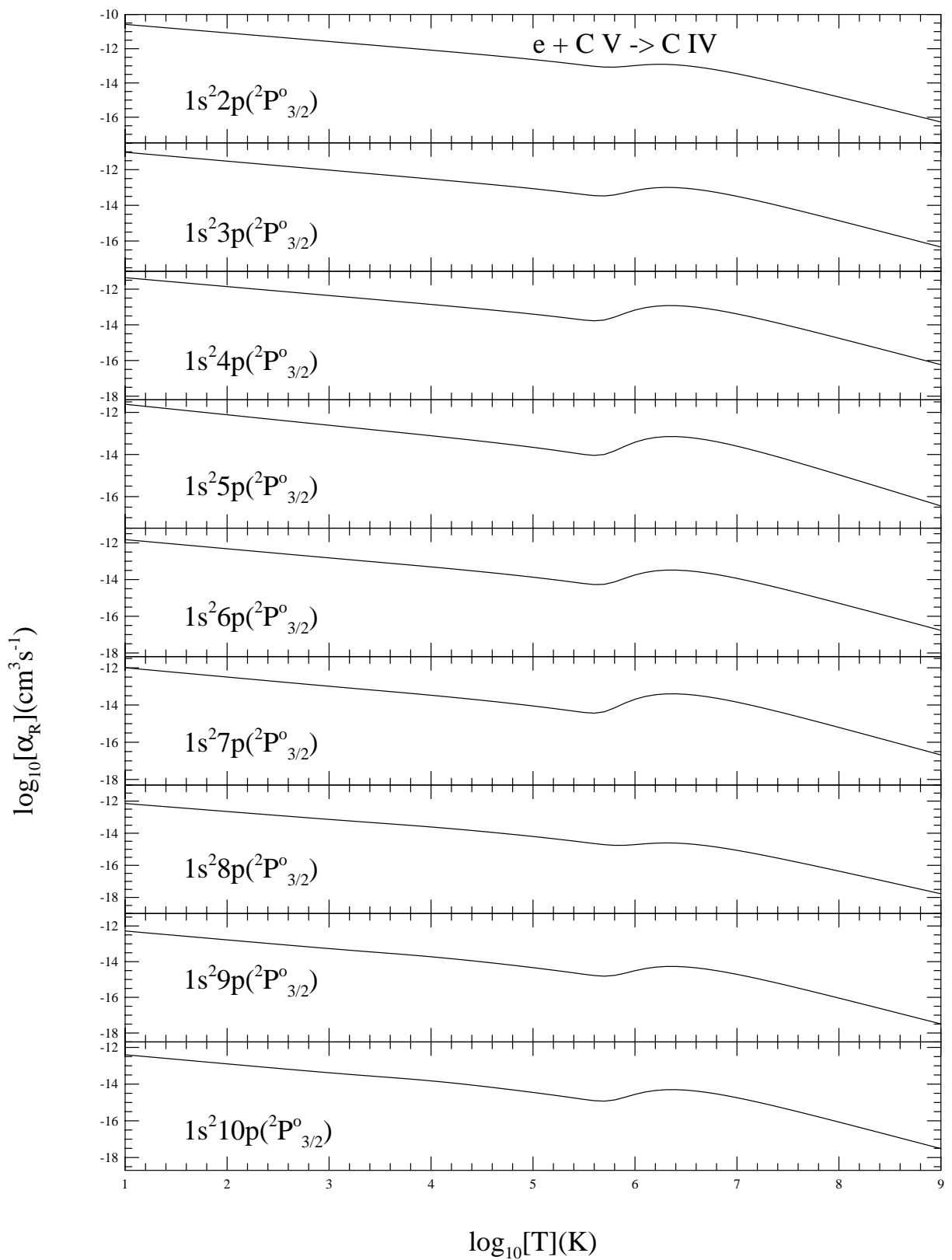

FIG. 6. - Level-specific recombination-rate coefficients of $e+\mathrm{C} \mathrm{v} \rightarrow \mathrm{C}$ IV into the ground and excited levels of the $1 s^{2} n p$ Rydberg series, $n \leq 10$

(Nahar \& Pradhan 1997). The earlier $L S$ rates overestimate the recombination rates at high temperatures. The dotted curve shows the rate coefficient computed using the Zhang et al. cross sections in a limited energy range with resonances, i.e., mainly DR. The DR rate by Shull \& Steenberg (1982; dot-dashed curve) agrees closely with the dotted curve. The dashed and the long-dashed curves are RR rates by Aldrovandi \& Pequignot (1973) and Verner \& Ferland (1996); they agree with the present rates at lower temperatures.

Table 2 presents the unified total BPRM recombination rate coefficients of $\mathrm{C}$ IV and $\mathrm{C} v$ averaged over a Maxwellian distribution.

\subsection{Level-specific Recombination Rate Coefficients}

Figure 8 shows the level-specific rate coefficients for the ground and the excited $n=2$ levels that are of considerable importance in X-ray spectroscopy as they are responsible for the formation of the $w, x, y, z$ lines from the four transitions $1 s^{2}\left({ }^{1} S_{0}\right) \leftarrow 1 s 2 p\left({ }^{1} P_{1}^{o}\right), 1 s 2 p\left({ }^{3} P_{2}^{o}\right), 1 s 2 p\left({ }^{3} P_{1}^{o}\right), 1 s 2 s\left({ }^{3} S_{1}\right)$. The present work is particularly relevant to the formation of these X-ray lines since recombination cascades from excited levels play an important role in determining the intensity ratios in coronal equilibrium and nonequilibrium plasmas (Pradhan 1985).

The rates in Figure 8 differ considerably from those by Mewe \& Schrijver (1978, hereafter MS) that have been widely employed in the calculation of X-ray spectra of He-like ions (e.g., Pradhan 1985). We compare with the direct (RR + DR) rates separately calculated by MS using approximate $Z$-scaled RR and DR rates for the individual $n=2$ levels of He-like ions. Their RR rates were from $Z$ scaled recombination rate of $\mathrm{He}^{+}$given by Burgess \& Seaton (1960); the $L S$-coupling data were divided according to the statistical weights of the fine-structure levels. Their DR rates were obtained using averaged autoionization 


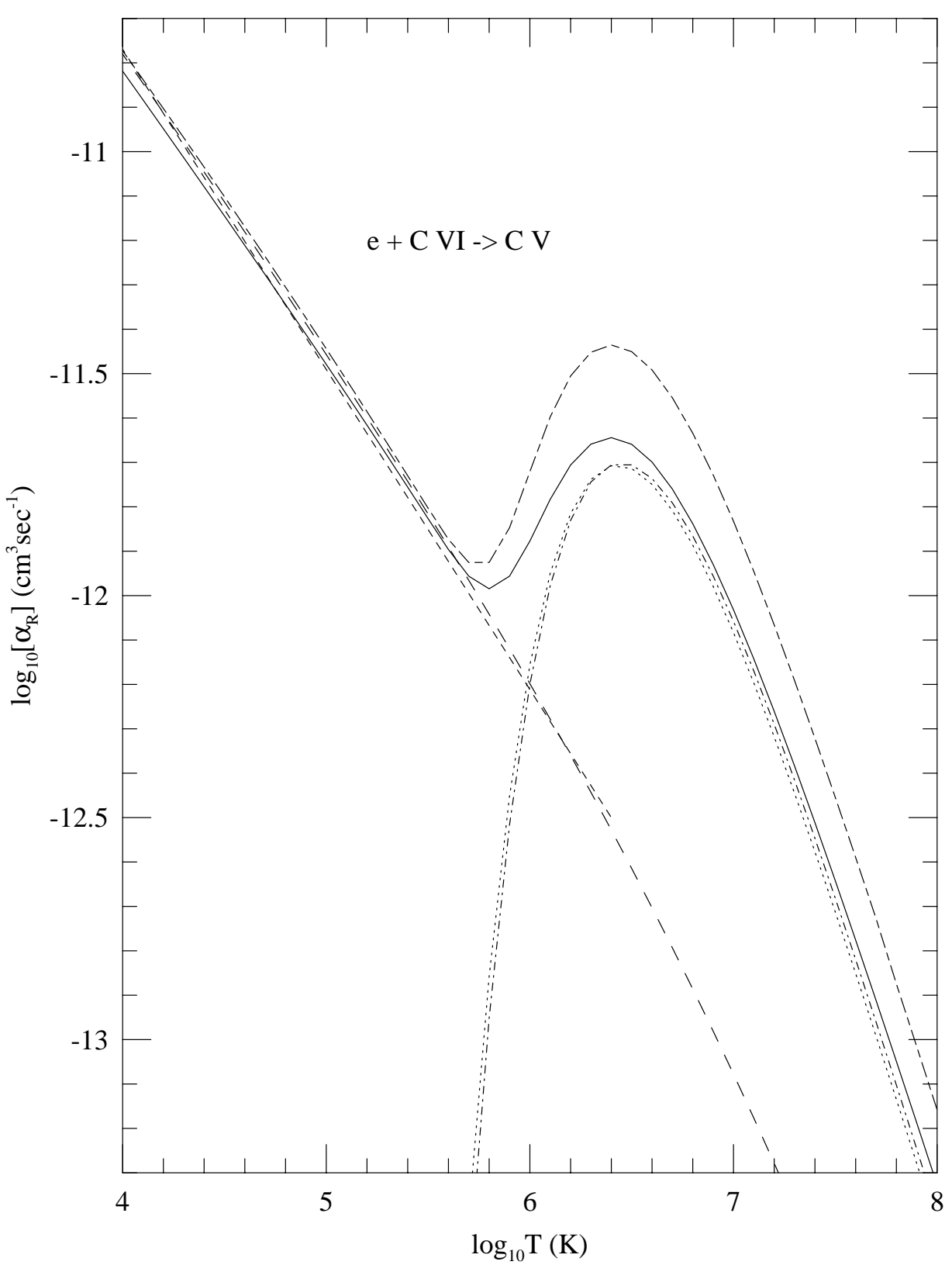

FIG. 7.- $(e+\mathrm{C} \mathrm{vI}) \rightarrow \mathrm{C} \mathrm{v}$; total unified rate coefficients: BPRM with fine structure - solid curve; LS coupling - short- and long-dashed curve, using Zhang et al. (1999) cross sections in the same range as experimental data—dotted curve; DR rates by Shull \& Steenberg (1982) - dot-dashed curve; RR rates by Aldrovandi \& Pequignot (1973) — short-dashed curve; Verner \& Ferland (1996) - long-dashed curve.

probabilities, $Z$-scaled from iron $(Z=26)$ and calculated with hydrogenic wave functions, together with radiative decay probabilities of the resonant $2 s 2 p, 2 p^{2},(2 p 3 s, 2 p 3 p$, $2 p 3 d$ ) levels, decaying to the final $n=2$ levels of He-like ions. The present work, on the other hand, includes DR contributions from all resonances up to $2 p n \ell$; $n \leq 10, \ell \leq n-1$. Figures 3 and 4 of Zhang et al. (1999) show the detailed photorecombination cross sections for these resonance complexes. But the present rate coefficients are much lower (Fig. 8). It is surprising that the MS rates are much higher than the present ones. If we consider, for example, the level-specific rate for the $1 s 2 s\left({ }^{3} S_{1}\right)$ level, the MS value includes contributions from only $2 s 2 p, 3 s 2 p, 3 p 2 p$ autoionizing levels. That the MS values overestimate the rates is also indicated by the fact that, at $\log T=6.4$ (the DR-peak temperature, Fig. 7), the sum of their individual $n=2$ level-specific rates is $1.5 \times 10^{-12} \mathrm{~cm}^{-3} \mathrm{~s}^{-1}$, compared with our unified total $\alpha_{R}=2.27 \times 10^{-12} \mathrm{~cm}^{-3} \mathrm{~s}^{-1}$ (Table 2; C v). That would imply that the MS rates for the $n=2$ levels alone account for $\frac{2}{3}$ of the total recombination $(\mathrm{RR}+\mathrm{DR})$ rate for $\mathrm{C} \mathrm{v}$; which is unlikely.

Figure 9 presents level-specific recombination-rate coefficients of $1 \operatorname{sns}\left({ }^{3} S\right)$ Rydberg series of C v levels to $n=10$. The features are similar to those of $\mathrm{C}$ IV. Although resolution of resonances in each cross section is very cumbersome, the sum of the level-specific rate coefficients, together with the DR contribution, agrees within a few percent of the total recombination-rate coefficient.

Recombination-cascade matrices may now be constructed for $\mathrm{C}$ IV and $\mathrm{C} v$, and effective recombination rates into specific levels obtained accurately using the direct recombination rates into levels with $n \leq 10, \ell \leq n-1$ 


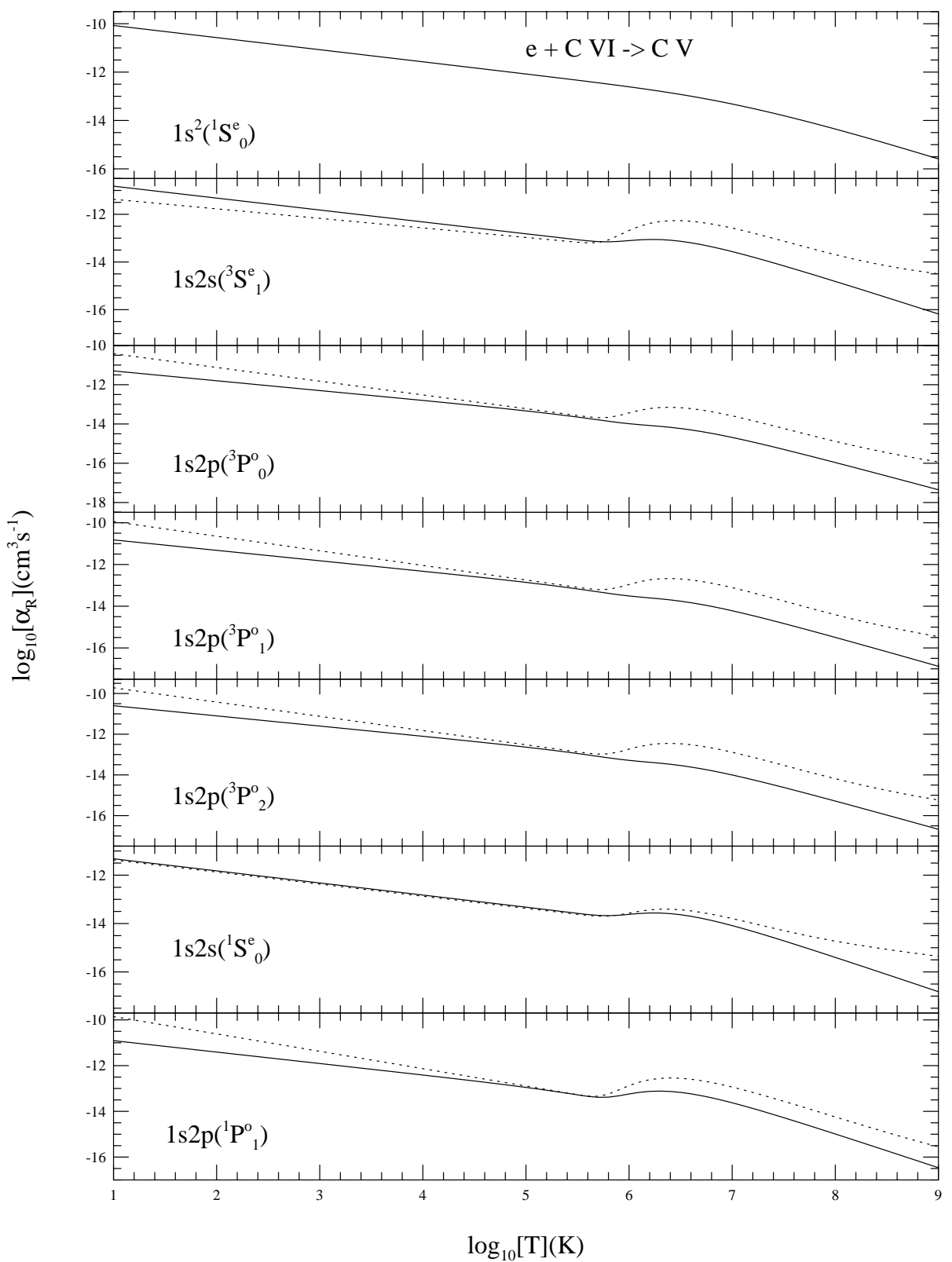

FIG. 8. -Direct, level-specific recombination-rate coefficients for $(e+\mathrm{C} \mathrm{vI}) \rightarrow \mathrm{C}$ v into the ground and the excited $n=2$ levels of $\mathrm{C}$ v-solid curve; Mewe \& Schrijver (1978) - dotted curve.

(Pradhan 1985). The present data is more than sufficient for extrapolation to the high- $n, \ell$ necessary to account for cascade contributions. Also needed are the radiative transition probabilities for all fine-structure levels of $\mathrm{C}$ IV and C $\mathrm{v}$, to the $n=10$ levels. They also have been calculated using the BPRM method and will be available shortly (S. N. Nahar, 2000, in preparation). These data will be similar to that for Fe XxIv and Fe XXv calculated earlier under the Iron Project (Nahar \& Pradhan 1999).

We discuss below some of the important atomic effects relevant to the present calculations in particular, and electron-ion recombination in general.

\subsection{Resolution and Radiation Damping of Resonances}

It is important to resolve near-threshold resonances $(v \leq 10)$ at an adequately fine energy mesh in order to (1) compute accurately their contribution to the rate coefficient or the averaged cross section and (2) to determine the radiative and autoionization rates through the fitting procedure referred to earlier (Sakimoto et al. 1990; Pradhan \& Zhang 1997; Zhang et al. 1999). Resonances narrower than the energy intervals chosen have very low autoionization rates and are mostly damped out; their contribution to (1) should be small.

\subsection{Interference between Resonant $(D R)$ and Nonresonant $(R R)$ Recombination}

In general, there is quantum mechanical interference between the resonant and the nonresonant components of the wave function expansion. Close-coupling photoionization calculations for strongly coupled near-neutral atomic systems cross sections overlap considerably between members of several Rydberg series of resonances that converge on to the excited, coupled target levels. 


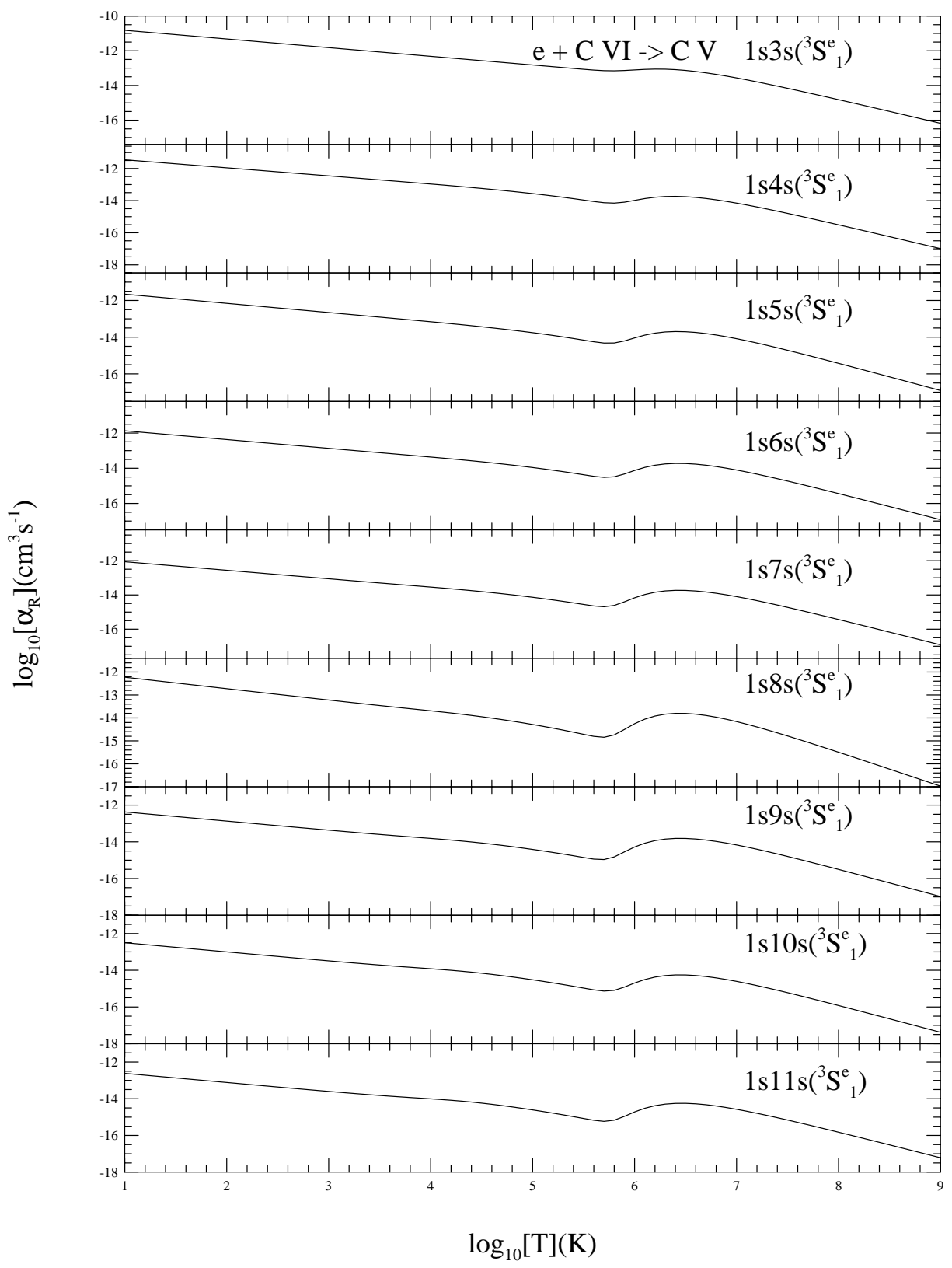

FIG. 9.-Level-specific recombination rate coefficients for $(e+\mathrm{C} \mathrm{VI}) \rightarrow \mathrm{C}$ v into the excited levels of the $1 s n s\left({ }^{3} S_{1}\right)$ Rydberg series, $n \leq 11$

Unified $(e+$ ion $)$ rates have been calculated for over 40 atoms and ions of astrophysical interest. The resonance structures in a number of these are extremely complicated and show considerable interference. The large number of photoionization calculations under the Opacity Project, for the ground state and the excited states (typically, a few hundred excited states for each atom or ion), show overlapping resonances to often dominate the cross sections. These are archived in the Opacity Project database TOPBASE and may be accessed on-line via the website http://heasarc.gsfc.nasa.gov or via the link from www.astronomy.ohio-state.edu/ pradhan). Furthermore, the excited-state cross sections of excited metastable states may exhibit even more extensive resonance structures than the ground state (e.g., in photoionization of $\mathrm{O}$ III; Luo et al. 1989). Strictly speaking, one needs to consider the partial photoionization cross sections for each of the three cases (dipole photoionization): $\Delta S=0, \Delta l=0, \pm 1$ in $L S$ coup- ling, or $\Delta J=0, \pm 1$ in BPRM calculations. Although these are not often tabulated or displayed, an examination thereof reveals that for near-neutrals each partial cross section shows overlapping resonances. As the ion charge increases, the resonances separate out, the interference decreases, and isolated resonance approximations may be used (Pindzola, Badnell, \& Griffin 1992; Zhang 1998).

Therefore, in general, we expect interference between the nonresonant $\mathrm{RR}$ and resonant $\mathrm{DR}$ recombination processes, particularly for many-electron systems where a separation between the two processes is unphysical and imprecise. Of course, experimentally, such a division is artificial and is not possible. For example, the recent experimental measurements on electron recombination with Fe XVIII to Fe XVII (Savin et al. 1997) clearly show the nearthreshold cross section to be dominated by the nonresonant recombination (RR) toward $E \rightarrow 0$, with superimposed resonance structures (the DR contribution) at higher energies. 


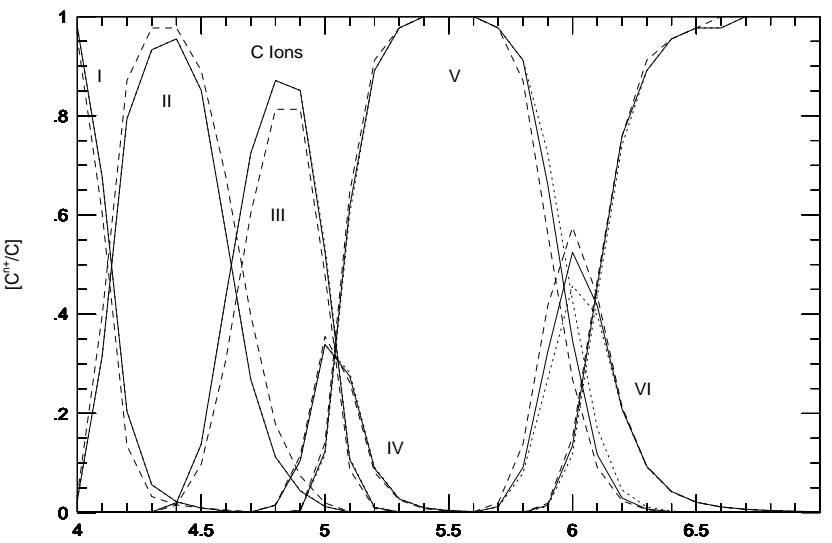

FIG. 10.--Ionization fractions of carbon in coronal equilibrium using the present recombination rates for $\mathrm{C}$ IV and $\mathrm{C} \mathrm{v}$-solid curve; using the LS coupling rates by Nahar \& Pradhan (1997) - dashed curve; ionization fractions from Arnaud \& Rothenflug (1985) - dotted curve.
In a recent work, Savin (1999) cited the work of Pindzola et al. (1992) to state that the effect of interferences is small (Pindzola et al. did not carry out CC calculations such as in the Opacity Project). Although this is not true in general, for highly ionized few-electron ions, one expects sufficient resonance separation so that an independent treatment of RR and DR may be accurate; such is the case for recombination with $\mathrm{H}$-like and He-like ions. The present unified treatment accounts for interference effects in an ab initio manner, and $(e+$ ion) rates have been calculated for over 40 atoms and ions of astrophysical interest (heretofore, in $L S$ coupling).

\subsection{Comparison with Experimental Data and Uncertainties}

Although experimental results are available for relatively few ions in limited energy ranges, and mostly for simple atomic systems such as the $\mathrm{H}$-like and He-like ions, they are very useful for the calibration of theoretical cross sections.

TABLE 2

Total Recombination Rate Coefficients, $\alpha_{R}(T)$, of C iv and C v

\begin{tabular}{|c|c|c|c|c|c|}
\hline \multirow{2}{*}{$\begin{array}{c}\log _{10} T \\
\text { (K) }\end{array}$} & \multicolumn{2}{|c|}{$\alpha_{R}\left(\mathrm{~cm}^{3} \mathrm{~s}^{-1}\right)$} & \multirow{2}{*}{$\begin{array}{c}\log _{10} T \\
\quad(\mathrm{~K})\end{array}$} & \multicolumn{2}{|c|}{$\alpha_{R}\left(\mathrm{~cm}^{3} \mathrm{~s}^{-1}\right)$} \\
\hline & $\mathrm{C}$ IV & $\mathrm{C} \mathrm{v}$ & & $\mathrm{C}$ IV & $\mathrm{C} \mathrm{v}$ \\
\hline $1.00 \ldots \ldots$ & $5.86 \mathrm{E}-10$ & $9.87 \mathrm{E}-10$ & $5.10 .$. & $1.32 \mathrm{E}-12$ & $2.84 \mathrm{E}-12$ \\
\hline $1.10 \ldots \ldots$ & $5.14 \mathrm{E}-10$ & $8.68 \mathrm{E}-10$ & $5.20 \ldots$ & $1.10 \mathrm{E}-12$ & $2.42 \mathrm{E}-12$ \\
\hline $1.20 \ldots \ldots$ & $4.51 \mathrm{E}-10$ & $7.62 \mathrm{E}-10$ & $5.30 \ldots$ & $9.17 \mathrm{E}-13$ & $2.06 \mathrm{E}-12$ \\
\hline $1.30 \ldots \ldots$ & $3.95 \mathrm{E}-10$ & $6.69 \mathrm{E}-10$ & $5.40 \ldots$ & $7.61 \mathrm{E}-13$ & $1.75 \mathrm{E}-12$ \\
\hline $1.40 \ldots \ldots$ & $3.45 \mathrm{E}-10$ & $5.88 \mathrm{E}-10$ & $5.50 \ldots$ & $6.34 \mathrm{E}-13$ & $1.49 \mathrm{E}-12$ \\
\hline $1.50 \ldots \ldots$ & $3.02 \mathrm{E}-10$ & $5.15 \mathrm{E}-10$ & $5.60 \ldots$ & $5.42 \mathrm{E}-13$ & $1.27 \mathrm{E}-12$ \\
\hline $1.60 \ldots$ & $2.63 \mathrm{E}-10$ & $4.51 \mathrm{E}-10$ & $5.70 \ldots$ & $5.11 \mathrm{E}-13$ & $1.11 \mathrm{E}-12$ \\
\hline $1.70 \ldots \ldots$ & $2.30 \mathrm{E}-10$ & $3.94 \mathrm{E}-10$ & $5.80 \ldots$ & $5.76 \mathrm{E}-13$ & $1.04 \mathrm{E}-12$ \\
\hline $1.80 \ldots \ldots$ & $2.01 \mathrm{E}-10$ & $3.45 \mathrm{E}-10$ & $5.90 \ldots$ & $7.53 \mathrm{E}-13$ & $1.11 \mathrm{E}-12$ \\
\hline $1.90 \ldots \ldots$ & $1.75 \mathrm{E}-10$ & $3.01 \mathrm{E}-10$ & $6.00 \ldots \ldots$ & $1.01 \mathrm{E}-12$ & $1.33 \mathrm{E}-12$ \\
\hline $2.00 \ldots \ldots$ & $1.52 \mathrm{E}-10$ & $2.63 \mathrm{E}-10$ & $6.10 \ldots \ldots$ & $1.28 \mathrm{E}-12$ & $1.65 \mathrm{E}-12$ \\
\hline $2.10 \ldots \ldots$ & $1.32 \mathrm{E}-10$ & $2.29 \mathrm{E}-10$ & $6.20 \ldots \ldots$ & $1.48 \mathrm{E}-12$ & $1.97 \mathrm{E}-12$ \\
\hline $2.20 \ldots \ldots$ & $1.15 \mathrm{E}-10$ & $2.00 \mathrm{E}-10$ & $6.30 \ldots \ldots$ & $1.57 \mathrm{E}-12$ & $2.19 \mathrm{E}-12$ \\
\hline $2.30 \ldots \ldots$ & $1.00 \mathrm{E}-10$ & $1.74 \mathrm{E}-10$ & $6.40 \ldots \ldots$ & $1.54 \mathrm{E}-12$ & $2.27 \mathrm{E}-12$ \\
\hline $2.40 \ldots \ldots$ & $8.68 \mathrm{E}-11$ & $1.52 \mathrm{E}-10$ & $6.50 \ldots \ldots$ & $1.42 \mathrm{E}-12$ & $2.19 \mathrm{E}-12$ \\
\hline $2.50 \ldots \ldots$ & $7.55 \mathrm{E}-11$ & $1.32 \mathrm{E}-10$ & $6.60 \ldots \ldots$ & $1.25 \mathrm{E}-12$ & $2.00 \mathrm{E}-12$ \\
\hline $2.60 \ldots \ldots$ & $6.53 \mathrm{E}-11$ & $1.15 \mathrm{E}-10$ & $6.70 \ldots$ & $1.05 \mathrm{E}-12$ & $1.74 \mathrm{E}-12$ \\
\hline $2.70 \ldots \ldots$ & $5.66 \mathrm{E}-11$ & $9.99 \mathrm{E}-11$ & $6.80 \ldots \ldots$ & $8.48 \mathrm{E}-13$ & $1.45 \mathrm{E}-12$ \\
\hline $2.80 \ldots \ldots$ & $4.90 \mathrm{E}-11$ & $8.67 \mathrm{E}-11$ & $6.90 \ldots \ldots$ & $6.69 \mathrm{E}-13$ & $1.18 \mathrm{E}-12$ \\
\hline $2.90 \ldots \ldots$ & $4.24 \mathrm{E}-11$ & $7.52 \mathrm{E}-11$ & $7.00 \ldots \ldots$ & $5.17 \mathrm{E}-13$ & $9.29 \mathrm{E}-13$ \\
\hline $3.00 \ldots \ldots$ & $3.66 \mathrm{E}-11$ & $6.52 \mathrm{E}-11$ & $7.10 \ldots \ldots$ & $3.93 \mathrm{E}-13$ & $7.20 \mathrm{E}-13$ \\
\hline $3.10 \ldots \ldots$ & $3.16 \mathrm{E}-11$ & $5.65 \mathrm{E}-11$ & $7.20 \ldots \ldots$ & $2.94 \mathrm{E}-13$ & $5.49 \mathrm{E}-13$ \\
\hline $3.20 \ldots \ldots$ & $2.73 \mathrm{E}-11$ & $4.90 \mathrm{E}-11$ & $7.30 \ldots$ & $2.18 \mathrm{E}-13$ & $4.13 \mathrm{E}-13$ \\
\hline $3.30 \ldots \ldots$ & $2.35 \mathrm{E}-11$ & $4.24 \mathrm{E}-11$ & $7.40 \ldots \ldots$ & $1.60 \mathrm{E}-13$ & $3.08 \mathrm{E}-13$ \\
\hline $3.40 \ldots \ldots$ & $2.02 \mathrm{E}-11$ & $3.67 \mathrm{E}-11$ & $7.50 \ldots \ldots$ & $1.17 \mathrm{E}-13$ & $2.28 \mathrm{E}-13$ \\
\hline $3.50 \ldots \ldots$ & $1.74 \mathrm{E}-11$ & $3.17 \mathrm{E}-11$ & $7.60 \ldots \ldots$ & $8.47 \mathrm{E}-14$ & $1.67 \mathrm{E}-13$ \\
\hline $3.60 \ldots \ldots$ & $1.50 \mathrm{E}-11$ & $2.74 \mathrm{E}-11$ & $7.70 \ldots \ldots$ & $6.12 \mathrm{E}-14$ & $1.23 \mathrm{E}-13$ \\
\hline $3.70 \ldots \ldots$ & $1.29 \mathrm{E}-11$ & $2.37 \mathrm{E}-11$ & $7.80 \ldots \ldots$ & $4.41 \mathrm{E}-14$ & $8.93 \mathrm{E}-14$ \\
\hline $3.80 \ldots \ldots$ & $1.10 \mathrm{E}-11$ & $2.04 \mathrm{E}-11$ & $7.90 \ldots \ldots$ & $3.17 \mathrm{E}-14$ & $6.49 \mathrm{E}-14$ \\
\hline $3.90 \ldots \ldots$ & $9.46 \mathrm{E}-12$ & $1.76 \mathrm{E}-11$ & $8.00 \ldots \ldots$ & $2.27 \mathrm{E}-14$ & $4.70 \mathrm{E}-14$ \\
\hline $4.00 \ldots \ldots$ & $8.10 \mathrm{E}-12$ & $1.52 \mathrm{E}-11$ & $8.10 \ldots \ldots$ & $1.62 \mathrm{E}-14$ & $3.40 \mathrm{E}-14$ \\
\hline $4.10 \ldots \ldots$ & $6.93 \mathrm{E}-12$ & $1.31 \mathrm{E}-11$ & $8.20 \ldots \ldots$ & $1.16 \mathrm{E}-14$ & $2.46 \mathrm{E}-14$ \\
\hline $4.20 \ldots \ldots$ & $5.92 \mathrm{E}-12$ & $1.13 \mathrm{E}-11$ & $8.30 \ldots \ldots$ & $8.27 \mathrm{E}-15$ & $1.77 \mathrm{E}-14$ \\
\hline $4.30 \ldots \ldots$ & $5.05 \mathrm{E}-12$ & $9.69 \mathrm{E}-12$ & $8.40 \ldots \ldots$ & $5.90 \mathrm{E}-15$ & $1.28 \mathrm{E}-14$ \\
\hline $4.40 \ldots \ldots$ & $4.30 \mathrm{E}-12$ & $8.33 \mathrm{E}-12$ & $8.50 \ldots \ldots$ & $4.20 \mathrm{E}-15$ & $9.20 \mathrm{E}-15$ \\
\hline $4.50 \ldots \ldots$ & $3.66 \mathrm{E}-12$ & $7.16 \mathrm{E}-12$ & $8.60 \ldots \ldots$ & $2.99 \mathrm{E}-15$ & $6.62 \mathrm{E}-15$ \\
\hline $4.60 \ldots \ldots$ & $3.10 \mathrm{E}-12$ & $6.15 \mathrm{E}-12$ & $8.70 \ldots \ldots$ & $2.13 \mathrm{E}-15$ & $4.76 \mathrm{E}-15$ \\
\hline $4.70 \ldots \ldots$ & $2.63 \mathrm{E}-12$ & $5.28 \mathrm{E}-12$ & $8.80 \ldots \ldots$ & $1.52 \mathrm{E}-15$ & $3.42 \mathrm{E}-15$ \\
\hline $4.80 \ldots \ldots$ & $2.22 \mathrm{E}-12$ & $4.53 \mathrm{E}-12$ & $8.90 \ldots \ldots$ & $1.08 \mathrm{E}-15$ & $2.46 \mathrm{E}-15$ \\
\hline $4.90 \ldots \ldots$ & $1.87 \mathrm{E}-12$ & $3.88 \mathrm{E}-12$ & $9.00 \ldots \ldots$ & $7.67 \mathrm{E}-16$ & $1.77 \mathrm{E}-15$ \\
\hline $5.00 \ldots \ldots$ & $1.57 \mathrm{E}-12$ & $3.32 \mathrm{E}-12$ & $\ldots$ & $\ldots$ & $\ldots$ \\
\hline
\end{tabular}


There is very good agreement with experimentally measured cross sections for electron recombination to C IV, C v, and O VII, as discussed in detail by Zhang et al. (1999). However, the energy range of experimental measurements is much smaller; the theoretical calculations are from $E=0$ to the very high energies necessary to obtain rate coefficients to $T=10^{9} \mathrm{~K}$. For recombination with $\mathrm{H}$ - and He-like ions, most (but not all) of the rates depend on relatively few of the low- $n$ complex of resonances in the low-energy region covered by experiments. In a recent work, Savin (1999) used the experimental cross sections for recombination with $\mathrm{C} \mathrm{V}$ to $\mathrm{C}$ IV, and $\mathrm{O}$ VIII to $\mathrm{O}$ VII, to obtain "experimentally derived" DR rate coefficients and compared those with several sets of theoretical data, including those in Papers I and II. (Strictly speaking, these rates include RR + DR contributions since the experimental cross sections always include both, and which the unified method aims to obtain.) Whereas the previous $L S$ coupling results for C IV (Paper I) were $43 \%$ higher than Savin's values, the results for O VII were in reasonable agreement, $\sim 20 \%$ higher, within estimated uncertainties.

The agreement between the unified rates and the experimentally derived DR rates is within our $10 \%-20 \%$ in the region (i.e., at temperatures) where DR contribution peaks, around $\log (T)=6.3$ for C IV (Fig. 2). As the reported experimental data did not extend to low energies, where the nonresonant RR contribution dominates, the unified rates are higher toward lower temperatures from the DR peak and deviate in a predictably straightforward manner from the DR-only results. The "experimentally derived" data by Savin (1999) was obtained with a limited energy range while our present results include a much larger range. Therefore our results are higher. The dotted curve in Fig. 4, which we also obtained over a limited energy range, agrees well with the dotted-long-dashed curve (Savin 1999). The differences are within our estimate of uncertainty in the present results, up to $20 \%$. Given that the experimental cross sections are also likely to be uncertain to about this range, the agreement seems remarkably good.

While the present unified cross sections can be compared directly with experimental measurements and the new rate coefficients are in good agreement with the experimentally derived DR rates for recombination with simple ions such as the $\mathrm{H}$ - and He-like ions, the experimental data may represent a lower bound on the field-free theoretical recombination rates owing to (1) high- $n$ and $\ell$ ionization reducing the "DR" peak and (2) limited energy range in experiments.

\subsection{General Features of $(e+$ ion) Recombination Rates}

The nonresonant " $R R$ " recombination peaks as $E, T \rightarrow$ 0 . This is caused by the dominant contribution from an infinite number of high Rydberg states of the $(e+$ ion $)$ system into which the slow moving electron may recombine. At low- $E$ and $T$, the total $\log _{10}\left[\alpha_{R}(T)\right]$ is shown as a straight line on the log-log scale because of the exponential Maxwellian damping factor $\exp (-E / k T)$. It is not entirely trivial to compute the low- $E$ and $T$ contributions (that we refer to as "high- $n$ top-up"). We adapt the accurate numerical procedure developed by Storey \& Hummer (1992) to calculate the $n, \ell$ hydrogenic photoionization cross section for $11 \leq n \leq \infty$ (Nahar 1996). It is noted that the high- $n$ top-up also represents the otherwise missing background contribution caused by high- $n$ resonant recombination (DR). Although this background contribution is small (negligibly so for the $\mathrm{H}$ - and He-like ions), it is included in the unified treatment.

The resonant contribution (DR) peaks at higher $E$ and $T$ corresponding to the excitation energies and temperatures of the strong dipole transition(s) in the core ion. This is the broad peak in $\alpha_{R}(T)$.

\subsection{Ionization Fractions of Carbon}

Figure 10 presents coronal ionization fractions of $C$ using the new BPRM recombination rates for $\mathrm{C}$ IV and $\mathrm{C} \mathrm{V}$ (solid lines). Also given are the results (dashed lines) from Arnaud \& Rothenflug (1985) and previous results (dotted lines) using $L S$ coupling rates from (Nahar \& Pradhan 1997). Differences with both sets of data may be noted for C IV, C V, C VI, and $\mathrm{O}$ VII. The most significant change (enhancement) is for $\mathrm{C}$ vI owing to the decrease in $\mathrm{C} \mathrm{V}$ recombination rate, and the new ionization fractions appear to be in better agreement with Arnaud \& Rothenflug (1985) than with the Nahar \& Pradhan (1997) results. Also discernible is the steeper fall-off in the $\mathrm{C} \mathrm{v}$ ionization fraction on the hightemperatures side.

\section{CONCLUSION}

New relativistic calculations are presented for the total, unified $(e+$ ion) rates coefficients for $\mathrm{C}$ IV and $\mathrm{C} \mathrm{V}$ of interest in X-ray astronomy. As the photorecombination cross sections in the dominant low-energy region have been shown earlier to be in very good agreement with experiments (Zhang et al. 1999), it is expected that the present rates should be definitive, with an uncertainty that should not exceed $10 \%-20 \%$.

The unified theoretical formulation and experimental measurements suggest both that the unphysical and imprecise division of the recombination process into radiative recombination (RR) and dielectronic recombination (DR) be replaced by nonresonant and resonant recombination since these are naturally inseparable.

Further calculations are in progress for oxygen $(\mathrm{O}$ VI and $\mathrm{O}$ VII) and iron (Fe XXIV and Fe XXV).

The available data includes

1. photoionization cross sections for bound finestructure levels of $\mathrm{C}$ IV and $\mathrm{C} v$ to $n=10$-both total and partial (into the ground level of the residual ion); and

2. total, unified recombination rates for $\mathrm{C}$ IV and $\mathrm{C} v$ and level-specific recombination-rate coefficients for levels to $n=10$.

All photoionization and recombination data are available electronically from the first author at nahar@, astronomy.ohio-state.edu. The total recombination-rate coefficients are also available from the Ohio State Atomic Astrophysics web site at www.astronomy.ohio-state.edu/ $\sim$ pradhan.

This work was supported in part by grants from NSF (AST-9870089) and NASA (NAG5-8423 and EL9-1013A). The computational work was carried out on the Cray T94 at the Ohio Supercomputer Center. 


\section{REFERENCES}

Aldrovandi, S. M. V., \& Pequignot, D. 1973, A\&A, 25, 137

Arnaud, M., \& Rothenflug, R. 1985, A\&AS, 60, 425

Badnell, N. R., Pindzola, M. S., \& Griffin, D. C. 1990, Phys. Rev. A, 41, 2422

Bell, K. L., Gilbody, H. B., Hughes, J. G., Kingston, A. E., \& Smith, F. J. 1983, J. Phys. Chem. Ref. Data, 12, 891

Bell, R. H., \& Seaton, M. J. 1985, J. Phys. B, 18, 1589

Berrington, K. A., Eissner, W., \& Norrington, P. H. 1995, Comput. Phys. Commun., 92, 290

Bregman, J. N., \& Harrington, J. P. 1986, ApJ, 309, 833

Burgess, A., \& Seaton, M. J. 1960, MNRAS, 121, 471

Crenshaw, D. M., \& Kraemer, S. B. 1999, ApJ, 521, 572

Gabriel, A. H. 1972, MNRAS, 160, 99

Hummer, D. G., Berrington, K. A., Eissner, W., Pradhan, A. K., Saraph, H. E., \& Tully, J. A. 1993, A\&A, 279, 298

Kilgus, G., et al. 1990, Phys. Rev. Lett., 64, 737

Kilgus, G., Habs, D., Schwalm, D., Wolf, A., Schuch, R., \& Badnell, N. R. 1993, Phys. Rev. A, 47, 4859

Laor, A., et al. 1994, ApJ, 420, 110

Luo, D., Pradhan, A. K., Saraph, H. E., Storey, P. J., \& Yu, Y. 1989, J. Phys. B, 22, 389

Mannervik, S., Asp, S., Broström, L., DeWitt, D. R., Lidberg, L., Schuch, R., \& Chung, K. T. 1997, Phys. Rev. A, 55, 1810

Martin, C., \& Bowyer, S. 1990, ApJ, 350, 242

Mewe, R., \& Schrijver, J. 1978, A\&A, 65, 99 (MS)

Nahar, S. N. 1996, Phys. Rev. A, 53, 2417
Nahar, S. N. 1999, ApJS, 120, 131

Nahar, S. N., \& Pradhan, A. K. 1994, Phys. Rev. A, 49, 1816 . 1995, ApJ, 447, 966

1997, ApJS, 111, 339 (Paper I)

1999, A\&AS, 135, 347

2000, Phys. Scr., 61, 675

The Opacity Project 1 \& 2. 1995-96, compiled by the Opacity Project team, with intro. by M. J. Seaton (Bristol, UK: Institute of Physics)

Pindzola, M. S., Badnell, N. R., \& Griffin, D. C. 1992, Phys. Rev. A, 46, 5725

Pradhan, A. K. 1985, ApJ, 288, 824

Pradhan, A. K., \& Shull, M. 1981, ApJ, 249, 821

Pradhan, A. K., \& Zhang, H. L. 1997, J. Phys. B, 30, L571

Sakimoto, K., Terao, M., \& Berrington, K. A. 1990, Phys. Rev. A, 42, 291

Savin, D. W. 1999, ApJ, 523, 855

Savin, D. W., et al. 1997, ApJ, 489, L115

Seaton, M. J. 1987, J. Phys. B, 20, 6363

Shull, J. M., \& Steenberg M. V. 1982, ApJS, 48, 95

Spitzer, L., Jr. 1990, ARA \&A, 28, 71

Spitzer, L., Jr., \& Fitzpatrick, E. L. 1993, ApJ, 409, 299

Storey, P. J., \& Hummer, D. G. 1992, Comput. Phys. Commun., 66, 129

Verner, D. A., \& Ferland, G. 1996, ApJS, 103, 467

Wolf, A., et al. 1991, Z. Phys. D Suppl., 21, 569

Zhang, H. L. 1998, Phys. Rev. A, 57, 2640

Zhang, H. L., Nahar, S. N., \& Pradhan, A. K. 1999, J. Phys. B, 32, 1459

Zhang, H. L., \& Pradhan, H. L. 1997, Phys. Rev. Lett., 78, 195 